\title{
Nephrology
}

Atala, A. c65

Aviv, A. e82

Bamri-Ezzine, S. e22

Baud, L. e13

Beck, L. e50

Bendayan, M. e22

Benöhr, P. e124

Blonski, M. e114

Cao, L.-C. e61

Chung, S.K. e89

Chung, S.S.M. e89

Dan, Q. e89

Danpure, C.J. e39

Delarue, F. e13
Durvasula, R. e114

Friedlander, G. e50

Furuse, Y. e100

Gardner, J.P. e82

Gingras, D. e22

Gleiter, C.H. e124

Goodver, P. e45

Griffin, S. e114

Habib, A.-M. e71

Harsch, S. e124

Hashimoto, N. e100

Haymann, J.-P. e13

Honeyman, T. e61

Iwamoto, I. e100

Jonassen, J.A. e61
Khan, S.R. e55
Kimura, M. e82
Knight, J.F. e71
Koh, C.J. e65
Krofft, R. e114
Lam, K.S.L. e89
Londoño, I. e22
Maekawa, M. e100
Nakao, A. e100
Nangaku, M. e8
Patterson, L.T. e109
Petermann, A.T. e114
Pippin, J. e114

Potter, S.S. e109

Prié, D. e50

Proksch, B. e124

Pusey, C.D. e71

Reynolds, J. e71

Saito, Y. e100

Sakurai, K. e100

Scheid, C.R. e61

Shankland, S.J. e114

Silve, C. e50

Skurnick, J. e82

Sraer, J.-D. e13

Suzuki, Y. e100

Tchakmakjian, L. e82
Torres, V.E. e1

Toshimori, K. e100

Toyama, Y. e100

Wahl, P. e31

Walters, G. e71

Wilson, P.D. e82

Wong, R.L.C. e89

$\mathrm{Wu}, \mathrm{H}$. e71

Wüthrich, R.P. e31

Yagui, K. e100

Yin, S. e89

Yuasa, S. e100

Zielke, H.R. e82

\section{Subject Index Vol. 98, 2004}

Advanced glycation end-products e89

African Americans e82

Aggregated immunoglobulins e13

$A G X T$ gene e 39

Alanine:glyoxylate aminotransferase e 39

Aldose reductase gene e89

Angiotensin II e124

Anoxia e8

B7RP-1 e 31

Bladder e65

Bone demineralization e50

Calcium e1

- oxalate e55

- _ kidney stones e39

cAMP e1

Caucasians e82

CDR3 spectratyping e71

Cell death e61

Cystinuria e45

Cytochemistry e22

Detachment of podocytes e114

Diabetes e22

Diabetic glomerulopathy e89

- nephropathy e89

- nephropathy, rats e114

Endocytosis e13
Enzyme replacement therapy e39

Erythropoietin e124

Experimental autoimmune glomerulonephritis e71

Fibroblast growth factor 23 e50

Fibrosis e8, e55

Gene expression e45, e61

- _ profile e109

Glomerular basement membrane e71

- epithelial cell e114

- _ cells e13

Glomerulus e100

Glycogen e22

HepG2 cells e124

Hyperphosphaturia e50

Hypertension e82

Hypoxia e124

Hypoxia-inducible factor e8

Hypoxia-responsive element e8

Inducible costimulator e31

Integrins e22

Interleukin-22 e124

Ischemia e8

Kidney e22

- development e109

- failure e8

- stone(s) e45, e61
-, therapeutic cloning and stem cells e65

LightCycler RT-PCR e124

Microarrays e109

Mitochondria e39, e61

Neonatal Fc receptor e13

Nephrocalcinosis e39

Nephrolithiasis e45, e50, e55

NPT2a e50

OLETF e100

Organogenesis e109

Oxalate(s) e39, e61

$D$-Penicillamine e45

Peroxisomes e39

Phosphate transport e50

Phospholipase A2 e61

Podocyte in diabetes e114

Polycystic kidney disease e1

Primary hyperoxaluria type 1 e 39

Raf e1

Ras e1

Reactive oxygen species e55

Reconstruction e65

Regenerative medicine e65

Renal growth e82

- podocytes e114

Renin angiotensin system e8

Smads e100
Sodium chloride e 82

Sodium-protein exchanger regulatory factor 1 e 50

T-cell receptor e71

Telomeres e82

TGF- $\beta_{1}$ e 89

Th1 e31

Th2 e31

Tissue engineering e65

Transforming growth factor- $\beta$ e100

Transgenic mouse e 89

Tubular epithelial cells e31

Tubules e8

Type IV collagen e89

Urethra e65

Urinary incontinence e65

Urinary tract e65

Urine, podocytes e114

Urokinase e13

Urolithiasis e 39

Vascular endothelial growth factor e8

Vasopressin V2 receptor antagonists e1

Vesicoureteral reflux e65

WKY rats e71

\section{KARGER}

(C) 2004 S. Karger AG, Basel

Fax + 41613061234

E-Mail karger@karger.ch

www. karger.com 